\title{
Impacto da automação no diagnóstico de infecções por micobactérias
}

\author{
Impact of automation in the diagnosis of mycobacterial infection
}

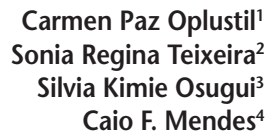

unitermos resumo

Micobactérias

A tuberculose é uma doença de importância mundial e há alguns anos, em muitos países, foi

Diagnóstico laboratorial

quase erradicada, mas, com o advento da Aids, novos casos da doença começaram a ocorrer, com o agravante do surgimento de cepas resistentes a diversos antimicrobianos.

Detecção automatizada

Concomitantemente a este aumento na incidência de tuberculose, as metodologias

diagnósticas apresentaram avanços consideráveis, e atualmente existem diversas metodologias

manuais ou automatizadas para um diagnóstico mais rápido das infecções por micobactérias.

Um dos sistemas semi-automatizados, o Bactec 460 (Becton Dickinson Diagnostic Systems,

Sparks, MD), é utilizado para detectar a presença de micobactérias em espécimes clínicos,

realizar testes de sensibilidade aos antimicrobianos e fazer a diferenciação entre micobactérias

do complexo tuberculosis e as não-pertencentes a este complexo, diminuindo o tempo do

processo em vários dias. $\mathrm{O}$ intuito deste trabalho foi verificar o impacto ocasionado com a

introdução de um sistema semi-automatizado na rotina do laboratório. No período de janeiro

a junho de 1995, foram processadas, pelo método tradicional de cultura em meio de

Lowenstein-Jensen (ㄴ)J), 326 amostras, das quais 39 (12\%) foram positivas, sendo 77\%

destas identificadas como Mycobacterium tuberculosis. Do total de amostras positivas para

M. tuberculosis, 29 (74,3\%) apresentaram um prazo de detecção superior a 30 dias. No mesmo

período, no ano de 1997, com a introdução do sistema semi-automatizado, foram processadas

340 amostras, das quais $50(14,7 \%)$ foram positivas, sendo $46 \%$ destas identificadas como

Mycobacterium tuberculosis. O tempo médio para detecção do crescimento das amostras

positivas para M. tuberculosis foi de 12 dias. A implantação do sistema automatizado para

culturas de micobactérias proporcionou um aumento no número de isolamento de diferentes

espécies, em diversos materiais clínicos, com diminuição no tempo de detecção, além de

oferecer maior segurança para os técnicos durante a realização destes procedimentos.

\section{abstract}

Tuberculosis is a disease of worldwide importance and several years ago in many countries the disease was almost eradicated. With the advent of Aids infection, new cases of the disease started to emerge with the appearance of multiple drug resistant strains. Together with the increase in

tuberculosis cases, diagnostic methodologies showed an advance and nowadays there are several manual and automated methodologies for the diagnosis of these infections. The Bactec 460 (Becton Dickinson Diagnostic Systems, Sparks, MD) system is used for the direct detection of mycobacteria in clinical specimens, for susceptibility testing against tuberculostatic drugs and differentiation of Mycobacterium tuberculosis complex from other species not of the complex, shortening the process in several days. In this study we evaluated the impact of the introduction of an automated system. In the period of January to June 1995, 326 specimens were processed using the traditional methodology of culture in Lowenstein-Jensen (LJ). Thirty-nine (12\%) were positive being $77 \%$ of these identified as M. tuberculosis. Of the total of 39 positive cultures for M. tuberculosis, 29

(74.3\%) showed a time for detection greater than 30 days. In the same period in the year of 1997 with the introduction of the semiautomated system, 340 specimens were processed of which 50

(14.7\%) were positive being $46 \%$ of these M. tuberculosis. The mean time for detection of positive cultures for M. tuberculosis was 12 days. The use of the automated system for culture of mycobacteria showed an increase in the number of different species isolated from different specimens, decreasing the time for detection and allowing a better and safe condition for technicians while executing theses procedures. 


\section{Introdução}

De todas as doenças infecciosas, a tuberculose é uma das que apresentam maior morbidade e mortalidade. Surtos de infecção por cepas multirresistentes e o aumento da incidência de infecções causadas por outras espécies de micobactérias impuseram a necessidade do desenvolvimento de novos métodos diagnósticos para estas infecções (6). Embora alguns progressos na detecção direta de micobactérias por metodologias moleculares estejam sendo realizados, as metodologias de cultura ainda são indispensáveis para o diagnóstico. O diagnóstico envolve diversas etapas, como pesquisa direta no material clínico, processamento do material para cultura do microrganismo, inoculação em diferentes meios de cultura, incubação em temperaturas adequadas, identificação das diferentes espécies por métodos bioquímicos convencionais e teste de avaliação da sensibilidade aos antimicrobianos mais freqüentemente utilizados no tratamento destas infecções (1-4). Os meios sólidos têm um papel importante nestas culturas, não somente por seu custo menor e eficácia, mas também pelo fato de propiciarem um reconhecimento rápido da eventual presença de culturas mistas. Entretanto o tempo médio para esta detecção é geralmente longo, e a taxa de isolamento é inferior àquela obtida com a utilização de meios líquidos. Apesar de ainda não existir um processo automatizado que realize todas as etapas já mencionadas, alguns equipamentos, meios ou metodologias desenvolvidos já melhoraram muito os procedimentos citados em termos de tempo e mão-de-obra empregados na sua realização.

Um dos sistemas semi-automatizados que realiza a cultura, diferenciação do complexo M. tuberculosis e o teste de avaliação da sensibilidade às drogas primárias é o Bactec 460 (Becton Dickinson Diagnostic Systems, Sparks, MD), introduzido na década de 70 e até hoje bastante utilizado quando são necessários resultados mais rápidos $(5,6)$. O sistema funciona como uma cabina de segurança, não permitindo que aerossóis eventualmente formados possam contaminar o ambiente. Uma luz ultravioleta dentro da cabina mantém um certo grau de esterilidade próximo às tampas dos fras$\cos$, à medida que as leituras são realizadas. Os frascos 12B, contendo a amostra e o meio de cultura 7H12, são colocados no equipamento para que possa ser feita a leitura. $\mathrm{A}$ leitura nada mais é do que a detecção da liberação de $\mathrm{CO}_{2}$ devido ao crescimento da micobactéria, marcado $c o m{ }^{14} \mathrm{C}$. A quantidade de $\mathrm{CO}_{2}$ liberado é medida e expressa em unidades de crescimento ( $\mathrm{Gl}$ - Growth Index). Amostras com contagens acima de $10 \mathrm{Gl}$ são consideradas prováveis amostras positivas. $\mathrm{O}$ sistema permite a diferenciação das micobactérias do complexo M. tuberculosis das nãopertencentes a este grupo pela utilização do teste do NAP ( $p$-nitro- $\alpha$-acetilamino- $\beta$-hidroxipropiofenona). Micobactérias pertencentes ao complexo $M$. tuberculosis são sensíveis a esta droga, e observamos assim a inibição do crescimento.

O intuito deste estudo foi verificar o impacto, no laboratório de microbiologia, da introdução de um sistema semi-automatizado no processo de detecção de amostras positivas para micobactérias.

\section{Material e métodos}

Para este estudo foram analisados, retrospectivamente, os resultados das culturas realizadas no Fleury - Centro de Medicina Diagnóstica durante os meses de janeiro a junho nos anos de 1995 e 1997. No ano de 1995 foram processadas pelo método manual tradicional 326 amostras consecutivas de diversos materiais clínicos. No mesmo período no ano de 1997, 340 amostras consecutivas foram processadas pelos métodos semi-automatizado e tradicional.

\section{Método tradicional (semeadura em meio de Lowenstein-Jensen)}

Dos diversos materiais biológicos que apresentavam contaminação com microbiota normal (escarro, lavado brônquico, urina, entre outros), foram realizados esfregaços em lâminas do material direto, para pesquisa de bacilos álcool-ácido-resistentes, pela coloração de ZiehlNeelsen. Em seguida, o material foi descontaminado pelo método de NALC ( $\mathrm{N}$-acetil-L-cisteína + $\mathrm{NaOH} 4 \%$ ). Após tratamento, do sedimento foram feitos novos esfregaços em lâmina para a coloração de Ziehl-Neelsen, e 0,2ml foi semeado em dois meios de Lowenstein-Jensen (LI)), um com antibióticos (anfotericina B, ácido nalidíxico e penicilina) e um sem antibióticos.

Os materiais sem contaminação com flora normal (liquor, líquido pleural, líquido sinovial) foram centrifugados, e o sedimento foi utilizado para preparar esfregaços para coloração de Ziehl-Neelsen e semeadura em dois LJ. Cada LJ foi semeado com 0,2ml do sedimento.

Os tubos foram incubados $37^{\circ} \mathrm{C}$ por cinco a sete dias com a tampa semi-aberta. Após este período, os tubos foram fechados e colocados na posição vertical, perma- 
necendo na estufa a $37^{\circ} \mathrm{C}$ por até oito semanas e sendo observados semanalmente. Em amostras com pesquisa direta positiva pela coloração de Ziehl-Neelsen, o meio de LJ foi incubado por 30 dias adicionais.

\section{Interpretação do crescimento}

Ao serem observadas colônias suspeitas no meio de L), foi confeccionado um esfregaço em lâmina, o qual foi corado com Ziehl-Neelsen para confirmação. A identificação da espécie foi feita por provas bioquímicas tradicionais $(3,4)$.

Após oito semanas de incubação, não havendo crescimento no LJ, as culturas foram consideradas negativas.

Para verificar as condições de crescimento do meio LJ), cada lote de meio foi testado com a cepa $M$. tuberculosis ATCC 25177. A esterilidade do meio foi verificada colocando-se o meio em aerobiose a $37^{\circ} \mathrm{C}$ por $48 \mathrm{~h}$ (7).

\section{Método automatizado (semeadura em meio 12B e meio de Lowenstein-Jensen)}

Os materiais que apresentavam microbiota normal foram tratados conforme descrito anteriormente no método tradicional. O frasco $12 \mathrm{~B}$ foi inoculado com $0,5 \mathrm{ml}$ do sedimento do material e $0,1 \mathrm{ml}$ de uma solução de antibióticos denominada Panta (polimixina, ácido nalidíxico, trimetoprima e anfotericina B). No meio de LJ $0,2 \mathrm{ml}$ do sedimento foi inoculado em apenas um tubo de meio. Para os materiais não-tratados, $0,5 \mathrm{ml}$ do material clínico foi inoculado em um frasco $12 \mathrm{~B}$ e $0,1 \mathrm{ml}$ da solução RF (reconstituting fluid) foi adicionado. Em outro tubo de LJ foi adicionado $0,2 \mathrm{ml}$ do material clínico não-tratado.

Amostras de sangue foram inoculadas diretamente apenas em frascos 13A, não sendo semeadas em meio de LJ.

Os frascos $12 \mathrm{~B}$ foram incubados a $37^{\circ} \mathrm{C}$ por um período máximo de oito semanas. As leituras foram realizadas no aparelho Bactec 460, duas vezes nas duas primeiras semanas e uma vez nas seis semanas seguintes. Os meios de LJ foram incubados e lidos conforme descrito no método tradicional. Em amostras com pesquisa direta positiva pela coloração de Ziehl-Neelsen, o meio de LJ foi incubado por 30 dias adicionais.

\section{Interpretação do crescimento}

Dos frascos $12 \mathrm{~B}$ que apresentaram $\mathrm{Gl} \geq 10$ foram confeccionadas lâminas para coloração pelo método de ZiehlNeelsen e uma amostra semeada em ágar chocolate para verificar possível contaminação com outros microrganis- mos. Os frascos $12 \mathrm{~B}$ confirmados como positivos pela coloração, sem crescimento nas placas de ágar chocolate após $24 \mathrm{~h}$ de incubação, foram incubados e lidos diariamente até o Gl alcançar níveis de 50 a 100, quando então foi realizado o teste do NAP para diferenciação do complexo M. tuberculosis. As micobactérias negativas para o teste do NAP foram identificadas por metodologia de sondas de DNA (GenProbe, San Diego, CA), complementadas por testes bioquímicos tradicionais quando necessário.

Para verificar as condições de crescimento do meio 12B cada lote de meio foi testado com a cepa M. tuberculosis ATCC 27294 (4).

\section{Resultados}

Neste estudo foram avaliados dois períodos diferentes, o primeiro, no qual o laboratório só utilizava o método tradicional de cultura de micobactérias, e o outro, no qual o sistema semi-automatizado Bactec 460 foi introduzido.

No período de 1995, 326 amostras foram processadas para cultura, das quais 39 (12\%) foram positivas, sendo que $30(77 \%)$ delas foram identificadas como M. tuberculosis. Das 30 amostras positivas para $M$. tuberculosis, $29(74,3 \%)$ apresentaram crescimento em um prazo superior a 30 dias de incubação, e em apenas sete $(23,3 \%)$ o crescimento ocorreu com menos de 30 dias de incubação. Neste período, as nove outras culturas positivas foram representadas por: quatro amostras com M. avium, duas com M. kansasii, duas com M. gordonae e uma com M. xenopi (Tabela 1).

No período de 1997 foram processadas 340 amostras para cultura, das quais $50(14,7 \%)$ foram positivas pelo Bactec 460, sendo que 23 (46\%) destas foram detectadas como M. tuberculosis. O prazo médio para detecção das amostras positivas pelo Bactec 460 foi de 12,7 dias, e das amostras positivas para M. tuberculosis foi de 12 dias. Com o sistema Bactec 460 foi detectado um número maior de diferentes espécies não-pertencentes ao complexo $M$. tuberculosis (Tabela 1). Das 23 amostras positivas para $M$. tuberculosis, cinco $(21,7 \%)$ apresentavam crescimento no frasco 12B, enquanto o meio LJ não mostrou crescimento ou a cultura contaminou com outro microrganismo, inviabilizando sua identificação no meio de LJ. Destas cinco amostras, duas apresentaram pesquisa direta negativa, e a detecção no frasco 12B foi feita em 18 e 24 dias, respectivamente (Tabela 2). Houve dois casos em que o frasco 
Tabela 1

Número de espécies de micobactérias isoladas pelos métodos tradicional e semiautomatizado Bactec 460 nos anos de 1995 e 1997, respectivamente

Método

Espécie

LJ (1995)

Número de cepas

isoladas (\% do total)

M. tuberculosis

$30(77)$

$2(5,1)$

$2(5,1)$

$4(10,2)$

M. avium

M. chelonae

M. scrofulaceum

M. xenopi

M. ulcerans

M. fortuitum

Total

\section{0}

0

$1(2,6 \%)$

0

0

$39(12)$
Bactec 460 (1997)

Número de cepas isoladas (\% do total)
$23(46)$
8 (16)
$2(4)$
$2(4)$
$4(8)$
$4(8)$
$2(4)$
$1(2)$
$1(2)$
$50(14,7)$

\section{Resultados das culturas positivas para $M$. tuberculosis no ano de 1997 pelo método}

Tabela 2 semi-automatizado Bactec 460 e no meio de cultura LJ

\begin{tabular}{lcccc}
\hline$N^{0}$ da amostra & $\begin{array}{c}\text { Material } \\
\text { clínico }\end{array}$ & $\begin{array}{c}\text { Resultado da } \\
\text { pesquisa direta }\end{array}$ & $\begin{array}{c}\text { Dias para detecção } \\
\text { pelo Bactec } 460\end{array}$ & $\begin{array}{c}\text { Dias para } \\
\text { detecção no LJ }\end{array}$ \\
3631 & LBA & + & 10 & $>30$ \\
6562 & Biópsia genital & - & 15 & 26 \\
660 & LCR & - & 9 & 21 \\
7276 & LCR & - & 13 & 15 \\
7544 & LBA & + & 4 & 37 \\
7563 & LCR & - & 18 & SC \\
8208 & Escarro & + & 7 & 21 \\
8370 & Escarro & + & 16 & $>30$ \\
8541 & LBA & + & 5 & 27 \\
8425 & Escarro & + & 5 & 30 \\
8479 & Escarro & + & 11 & Cont. \\
8947 & Escarro & + & 11 & SC \\
9637 & Urina & - & 6 & 23 \\
512 & Escarro & + & 2 & $>30$ \\
1093 & Escarro & + & 24 & SC \\
1253 & Escarro & - & 23 & 39 \\
1507 & Escarro & - & Cont. & 21 \\
205 & LBA & - & 24 & SC \\
3649 & LBA & - & 20 & 48 \\
4736 & Urina & + & 9 & 26 \\
6070 & LCR & - & 6 & 25 \\
7463 & Linfonodo & + & 16 & 13 \\
8494 & Escarro & - & 6 & 23 \\
\hline
\end{tabular}

LCR: liquor; LBA: lavado broncoalveolar; (-): negativo; (+): positivo; Cont.: cultura contaminada com outros microrganismos, o que inviabiliza o prosseguimento da cultura; L): Lowenstein-Jensen; SC: sem crescimento. 
12B se contaminou, inviabilizando a continuação da cultura, mas o meio de LJ apresentou-se positivo para M. tuberculosis.

A amostra número 512, de escarro, foi detectada como positiva pelo Bactec após dois dias de incubação, e o LJ apresentou crescimento com mais de 30 dias de incubação. Algo semelhante ocorreu com a amostra número 7544 de lavado broncoalveolar, na qual o período de incubação para detecção dos positivos foi de quatro dias.

A Tabela 3 relaciona as outras espécies de micobactérias que não as do complexo $M$. tuberculosis que foram detectadas, e verificamos que, das 27 culturas positi- vas no meio $12 \mathrm{~B}$, apenas seis foram isoladas concomitantemente no meio LJ. Uma amostra de pele ( $n^{\circ}$ 3575) foi detectada como positiva apenas no meio líquido em um período de tempo muito curto, e a identificação revelou ser M. ulcerans, que é uma micobactéria de crescimento lento.

\section{Discussão}

Apesar de ser uma doença antiga em nosso meio, os conceitos dentro do laboratório devem ser modernizados para acompanhar as necessidades de diagnóstico e de tratamento. Os pontos mais importantes que devem ser atingidos

Resultados das culturas positivas para outras micobactérias que não as do complexo $M$. tuberculosis, Tabela 3 no ano de 1997 pelo método semi-automatizado Bactec 460 e por cultura em meio de IJ

\begin{tabular}{|c|c|c|c|c|c|}
\hline $\begin{array}{l}\mathrm{N}^{0} \text { da } \\
\text { amostra }\end{array}$ & Espécie & $\begin{array}{l}\text { Material } \\
\text { clínico }\end{array}$ & $\begin{array}{l}\text { Resultado da } \\
\text { pesquisa direta }\end{array}$ & $\begin{array}{c}\text { Dias para detecção } \\
\text { pelo Bactec } 460\end{array}$ & $\begin{array}{c}\text { Dias para detecção } \\
\text { no LJ }\end{array}$ \\
\hline 2739 & M. kansasii & Escarro & + & 5 & $>30$ \\
\hline 2922 & M. xenopi & LBA & - & 20 & Cont. \\
\hline 3427 & M. gordonae & Urina & - & 37 & SC \\
\hline 3496 & M. gordonae & Urina & - & 14 & SC \\
\hline 3539 & M. gordonae & Escarro & - & 22 & $>30$ \\
\hline 3575 & M. ulcerans & Pele & + & 6 & $S C^{*}$ \\
\hline 4134 & M. fortuitum & Escarro & - & 25 & $>30$ \\
\hline 4708 & M. chelonae & Escarro & - & 8 & SC \\
\hline 4071 & M. avium & Sangue & NR & 17 & NR \\
\hline 4693 & M. avium & Sangue & NR & 11 & NR \\
\hline 5510 & M. avium & Linfonodo & + & 4 & $>30$ \\
\hline 6409 & M. chelonae & Fragmento ósseo & - & 7 & SC \\
\hline 7285 & M. gordonae & Urina & - & 12 & SC \\
\hline 8956 & M. avium & Fezes & + & 5 & 7 \\
\hline 8959 & M. avium & Sangue & NR & 8 & NR \\
\hline 9870 & M. kansasii & Escarro & - & 33 & SC \\
\hline 298 & M. gordonae & Urina & - & 17 & SC \\
\hline 1641 & M. scrofulaceum & Escarro & + & 18 & $S C^{*}$ \\
\hline 2381 & M. scrofulaceum & Escarro & - & 12 & SC \\
\hline 2815 & M. gordonae & Escarro & - & 10 & SC \\
\hline 3459 & M. scrofulaceum & Escarro & - & 13 & SC \\
\hline 3608 & M. scrofulaceum & Escarro & - & 14 & SC \\
\hline 4664 & M. chelonae & Escarro & - & 5 & Cont. \\
\hline 5046 & M. gordonae & Escarro & - & 17 & 43 \\
\hline 5213 & M. xenopi & Escarro & - & 14 & SC \\
\hline 5995 & M. chelonae & Escarro & - & 8 & SC \\
\hline 7055 & M. gordonae & Urina & - & 19 & Cont. \\
\hline
\end{tabular}

LBA: lavado broncoalveolar; NR: não-realizado; Cont.: cultura contaminada com outros microrganismos, o que inviabiliza o prosseguimento da cultura; (-): negativo; (+): positivo, SC: sem crescimento. *Amostras com pesquisa direta positiva sem crescimento no L f foram incubadas por mais 30 dias e não apresentaram crescimento. 
quando se pretende fazer o diagnóstico de infecções por micobactérias são: liberação do resultado do exame direto no prazo máximo de $24 \mathrm{~h}$ após o recebimento do material no laboratório; detecção dos bacilos álcool-ácido-resistentes em até no máximo 14 dias; realização da identificação no máximo entre 17 a 21 dias; e disponibilização para o médico do resultado da sensibilidade da micobactéria aos tuberculostáticos dentro de no máximo 28 dias (6).

O sistema Bactec 460 é utilizado para detecção, identificação e teste de avaliação da sensibilidade às drogas, mostrando resultado rápido quando comparado com os métodos tradicionais (5).

Este estudo consistiu em verificar o quanto a metodologia semi-automatizada implantada em nosso laboratório melhorou o diagnóstico das infecções por micobactérias. Procuramos estudar a mesma época, em diferentes anos, para que não houvesse interferência de fatores como diminuição ou aumento do número de exames determinados por fatores de sazonalidade.

Apesar de ter havido uma diferença pequena no número total de amostras processadas nos dois anos (326/ 1995 e 340/1997), no período de 1995 o número total de amostras positivas foi também proporcionalmente menor (12\%) do que no ano de 1997 (14\%), o que eventualmente pode ser explicado pela maior sensibilidade que o meio líquido apresenta em relação ao meio sólido (5). Resultados semelhantes foram encontrados por Presslich et al. (8), quando, de 802 amostras analisadas, $13,6 \%$ foram positivas pelo sistema Bactec e 11,3\% pelo sistema convencional.

O número de diferentes espécies isoladas no período de 1997 em relação ao período de 1995 foi também maior, o que mostra que o método automatizado propicia o meIhor desenvolvimento de micobactérias outras que não as do complexo M. tuberculosis (Tabela 1). Já a positividade para M. tuberculosis no ano de 1995 foi levemente maior (9,2\%) em relação ao ano de 1997 (6,8\%), o que poderia ser atribuído ao fato de que a incidência de infecções por M. tuberculosis teve uma ligeira diminuição devido ao uso dos medicamentos contra o HIV, além do aumento das infecções por outras espécies de micobactérias.

O tempo de detecção das amostras positivas pelo Bactec 460 foi em média de 12,7 dias, variando de dois a 37 dias. Uma amostra detectada com dois dias de incubação foi identificada como M. tuberculosis, demonstrando que, apesar de ser uma micobactéria de crescimento em geral lento, o sistema é bastante eficiente. O tempo médio de detecção de $M$. tuberculosis (12 dias) encontrado em nosso trabalho foi muito semelhante aos relatados por Morgan et al. (9) (13,7 dias), Presslich et al. (8) (15 dias) e Svecova (10) (12,9 dias). Outras micobactérias consideradas de crescimento lento também foram detectadas em um período menor de tempo pelo Bactec 460. Uma das amostras em que foi isolado $M$. kansasii apresentou pesquisa positiva, O 12B mostrou crescimento após cinco dias de incubação e o meio de LJ somente mostrou crescimento após mais de 30 dias de incubação. Já uma amostra de pele que apresentou crescimento de M. ulcerans, uma micobactéria de crescimento lento que apresenta a característica de crescer melhor quando incubada a $30^{\circ} \mathrm{C}$, foi detectada apenas no frasco 12B. Nesta amostra, o meio de LJ semeado ao mesmo tempo e incubado a $37^{\circ} \mathrm{C}$ não mostrou crescimento. Isto mostra que o meio $12 \mathrm{~B}$, apesar de não ter sido incubado na temperatura ideal para a espécie em questão, apresenta excelentes condições para seu isolamento.

As amostras de M. avium isoladas de amostras de sangue foram detectadas em média após 12 dias de incubação, variando de oito a 17 dias, mas não foram inoculadas em paralelo em meio de LJ, uma vez que eram colhidas diretamente no frasco, o que nos impede de fazer uma comparação direta. Já no ano de 1995, não foi detectada amostra de sangue positiva para micobactérias, apenas uma amostra de medula óssea em que foi isolado M. avium (Tabela 3). Estes resultados sugerem que a utilização de um meio líquido para este tipo de material clínico é extremamente importante.

Pelos resultados apresentados em 1997, o método tradicional não apresentou crescimento de $M$. tuberculosis em cinco amostras; destas, apenas duas apresentavam pesquisa direta negativa, o que poderia levar a pensar que, quando o número de bacilos na amostra é pequeno, o sistema Bactec 460 apresenta maior sensibilidade (Tabela 2). Já para as outras três amostras em que a pesquisa direta foi positiva, uma apresentou contaminação do meio LJ, o que inviabilizou a continuação da cultura, uma vez que as bactérias contaminantes alteram o meio. As outras duas eram amostras de escarro que não teriam por que não ter apresentado crescimento no meio de LJ, sendo que uma possível explicação seria que o meio líquido proporciona melhores condições para isolamento de micobactérias.

Foi grande também o número de amostras em que foram isoladas outras micobactérias que não as do complexo M. tuberculosis apenas no frasco $12 \mathrm{~B}$, sendo que a pesquisa inicial foi negativa. Isto poderia sugerir uma contaminação do meio 12B, mas em geral M. kansasii, 
M. avium e $M$. scrofulaceum não são considerados contaminantes de laboratório. O que sabemos é que em alguns materiais clínicos, como urina, M. gordonae pode ser encontrado como saprófita, em pequenas quantidades, e somente uma metodologia mais sensível que utiliza um meio líquido acaba detectando a sua presença.

Uma das melhores medidas para se prevenir a disseminação da tuberculose é o diagnóstico rápido e preciso da doença. Sabe-se também, por diversos estudos, que o meio líquido é mais sensível para a detecção do crescimento de micobactérias, mas algumas vezes apresenta um índice de contaminação mais elevado do que o meio sólido, e até materiais habitualmente estéreis como liquor estão sujeitos a contaminação devido ao procedimento realizado na manipulação no momento da inoculação. Neste estudo verificamos que o método Bactec 460 é sem dúvida melhor que o de cultura em meio sólido, mas não deve ser o único método empregado no laboratório para o diagnóstico. O sistema mostrou também que, além de aumentar a positividade, houve considerável diminuição do prazo de detecção das amostras positivas. As desvantagens do método Bactec 460 estão no custo elevado do meio de cultura; em o meio utilizado conter material radioativo; na utilização de um equipamento para realizar a leitura das culturas; e em estas leituras serem feitas introduzindo-se agulhas no frasco. Outro ponto importante a ser considerado é que os meios líquidos, em geral, apresentam um índice maior de contaminação que os meios sólidos.

Devemos salientar que todos os procedimentos para detecção de infecções por micobactérias devem ser realizados em salas especiais com exaustão apropriada e por uma equipe técnica com treinamento especializado.

\section{Referências}

I. Eisenstadt, J. et al. Mycobacterium tuberculosis and other nontuberculous mycobacteria. In: Mahon, C.R. \& Manuselis, G. Textbook of diagnostic microbiology. Philadelphia: W.B. Saunders Company, 1995. p. 636-76.

2. Heifets, L.B. Clinical Mycobacteriology. In: Clinic in laboratory medicine. Philadelphia:WB Saunders Company, 1996. v. 16.

3. Kent, P.T. \& Kubrica, G. Public health mycobacteriology. A guide for the level III laboratory. Atlanta: Centers for Disease Control and Prevention, 1985.

4. Master, R.N. Mycobacteriology. In: Isenberg, H. Clinical microbiology procedures handbook. Washington: ASM, I 992. v. I.

5. Roberts, G.D. et al. Evaluation of Bactec radiometric method for recovery of mycobacteria and drug susceptibility testing of Mycobacterium tuberculosis from acid-fast smear positive specimens. J. Clin. Microbiol., 18: 689-96, 1983.

6. Tenover, F.C. et al. The resurgence of tuberculosis: is your laboratory ready? J. Clin. Microbiol., 31: 767-70, 1993.
7. Sewell, D.L. Quality assurance, quality control, laboratory records, and water quality. In: Isenberg, $\mathrm{H}$. Clinical microbiology procedures handbook. Washington: ASM, 1992. v. 2.

8. Presslich, J. et al. The Bactec-System in the diagnosis of tuberculosis: comparison of a conventional and the radiometric method (Bactec) for culturing, differentiation and susceptibility testing of mycobacteria. Zentralbl Bakteriol Mikrobiol Hyg., 270(4): 487-9I, 1989

9. Morgan, M.A. et al. Comparison of a radiometric method (Bactec) and conventional culture media for recovery of mycobacteria from smear-negative specimens. J. Clin. Microbiol., 18: 384-8, 1983.

10. Svecova, Z. Detection of mycobacteria using the Bactec radiometry method and standard culture methods. Epidemiol. Mikrobiol Imunol., 46: 27-9, 1997. 